\title{
MECHANICAL AND ELECTRICAL PROPERTIES OF A NiTi SHAPE MEMORY ALLOY
}

\author{
G. AIROLDI ${ }^{*, * *}$, T. RANUCCI ${ }^{* *}$ and G. RIVA* \\ *I.N.F.M., Dipartimento di Fisica, Universita' di Milano, Via Celoria 16, I-20133 Milano, Italy \\ **I.T.M.-C.N.R., Area della Ricerca di Milano, Via Bassini 15, I-20133 Milano, Italy
}

\begin{abstract}
Mechanical properties of shape memory NiTi alloys have been widely investigated: though a reasonable understanding of the micromechanisms underlying macroscopic behaviour is known, these sometimes can hardly be inferred from macroscopic stressstrain curves. Electrical Resistance (ER) measurements, extensively used to investigate thermal martensite, are here performed on NiTi alloys under stress state. Attention is here focused on the mechanical and electrical behaviour of a NiTi alloy in one start single phase:

-in martensitic phase, at $\mathrm{T}<\mathrm{M}_{f}$ or within the hysteresis cycle;

-in parent phase, at $T>A_{f}$ or within the hysteresis cycle.

ER, sensitively affected by electronic structure modifications, lattice structure defects, residual impurities, can profitably be exploited to enlighten similar macroscopic stress-strain behaviour. ER results here obtained allow to discriminate different active micromechanisms, specifically at a same temperature within the hysteresis cycle where similar stressstrain curves are obtained.
\end{abstract}

\section{1.- Introduction}

The mechanical properties of NiTi Shape Memory Alloys (SMA) have been widely investigated, and are fairly well understood through a considerable amount of fundamental and applied research, as reviewed in (1). It is well known that the SMA mechanical response of a NiTi alloy to an applied stress is impressively temperature dependent, being related to the distinct micromechanisms involved in the deformation processes of either the high temperature B2 phase or the B19' martensite.

In full martensitic phase $\left(T<M_{f}\right)$, uniaxial loading gives rise to an elastic deformation range followed, at a temperature dependent pseudoyielding, by a smooth transition to the nearly constant stress variant re-orientation domain. Strains up to 88 , frozen in martensitic polycrystalline NiTi, can afterwards be recovered by 
heating in full B2 phase (shape memory effect).

In full parent phase, in the temperature domain $A_{f}, M_{d}$, an increasing stress induces an elastic deformation until pseudo-yielding takes place: at a further small stress increase, martensite is stressinduced at nearly constant stress (pseudoelasticity).

Inside the hysteresis cycle both processes can be present at the same time and it may be hard to distinguish the underlying deformation mechanisms from macroscopic stress-strain behaviour.

Electric Resistance (ER) measurements have been traditionally adopted to investigate martensitic transformations: as a matter of fact, ER is sensitively affected by electronic band structure modifications. Moreover, as ER depends also upon lattice structure defects, residual impurities, it can profitably be exploited to enlighten different microstructuctural contributions in similar macroscopic stress-strain behaviours.

Detection of ER simultaneously to stress-strain tensile test has been here undertaken on NiTi specimens in one start single phase, either martensitic B19' phase or parent B2 phase, in order to clarify the different deformation active micromechanisms.

\section{2.-Experimental}

NiTi rod specimens (diam. $3 \mathrm{~mm}$, lenght $100 \mathrm{~mm}$ ) were submitted to a preliminar $480^{\circ} \mathrm{C}(1 \mathrm{~h}+\mathrm{WQ})$ ageing treatment.

ER measurements were performed by means of a Digital Converter Ohmeter 1701b ("four wires method"), interfaced to an Olivetti PC: acquisition and data handling were carried out by software packages "ad hoc" developed.

Tensile tests were performed by means of a material testing equipment Instron 8032 provided with a thermostatic chamber which allowed to investigate the temperature range $-80^{\circ} \mathrm{C},+100^{\circ} \mathrm{C}$. Tensile tests were performed in strain control mode at $\operatorname{strain}(\epsilon)$ rate $d \epsilon / d t=0.018 / \mathrm{sec}$.

\section{3.-Results}

ER measurements were preliminarly performed as a function of temperature in order to check the transformation temperatures. Fig.1 shows a typical ER versus temperature curve: the rise of resistance on cooling before the main peak (corresponding to $M_{S}$ ) is related to the parent-to-Rhombohedral(R) phase transition(2-3). The detected transformation temperatures of the transitions were, on cooling, $\mathrm{T}_{r}=20^{\circ} \mathrm{C}, \mathrm{M}_{\mathrm{S}}=-14^{\circ} \mathrm{C}, \mathrm{M}_{f}=-65^{\circ} \mathrm{C}$, where $\mathrm{T}_{r}$ corresponds to the $\mathrm{B} 2->\mathrm{R}$ onset temperature; on heating, $A_{S}=15^{\circ} \mathrm{C}, A_{f}=38^{\circ} \mathrm{C}$.

Tensile tests were performed either outside or inside the hysteresis cycle at the following $T_{i}(i=1,2,3,4)$ temperature:

1) at $T_{1}=-80^{\circ} \mathrm{C}<M_{f}$ and at $T_{2}=+5^{\circ} \mathrm{C}<A_{S}$ in full B19' phase;

2) at $T_{3}=+5^{\circ} \mathrm{C}<T_{r}$, where $M_{S}<T_{3}<T_{r}$, in R-phase;

3) at $\mathrm{A}_{\mathrm{F}}<\mathrm{T}_{4}=+50^{\circ} \mathrm{C}$ in full B2 phase;

with the simultaneous detection of ER during loading. 


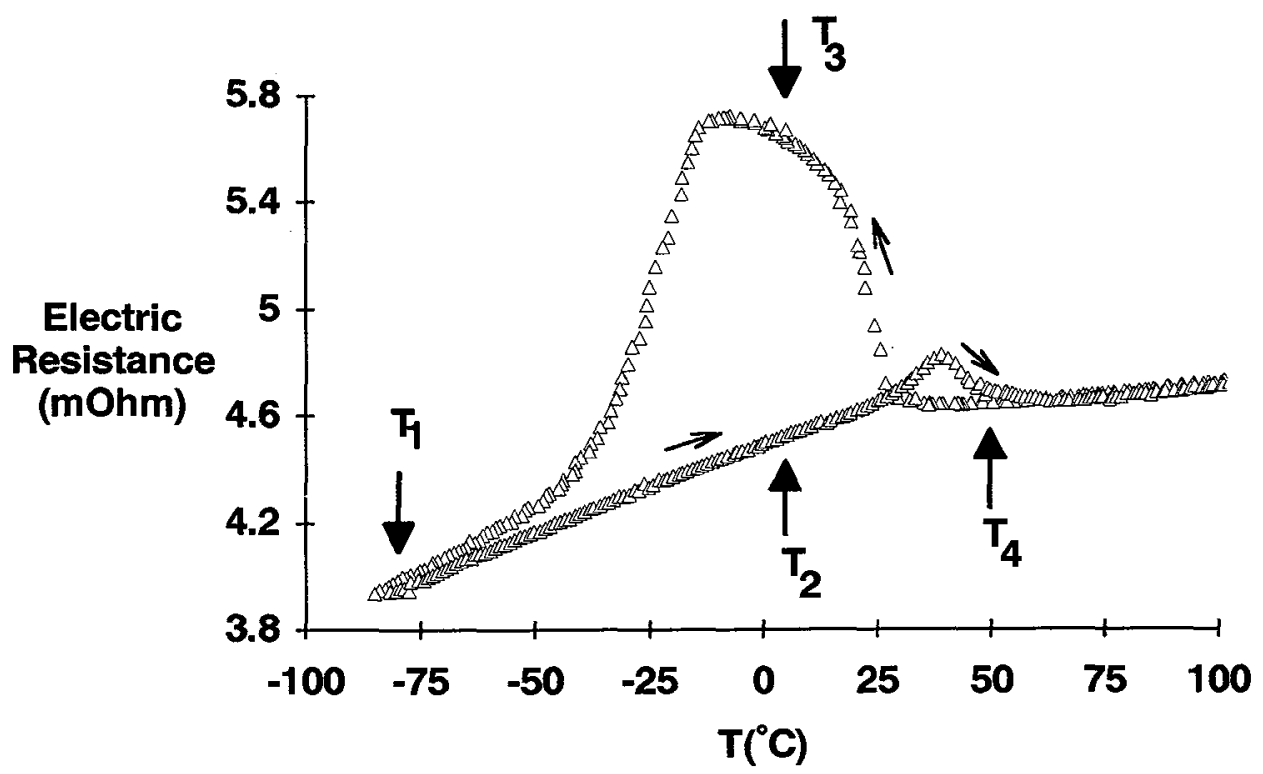

Fig.1 - Electric Resistance vs. Temperature curve of the examined NiTi specimens without applied stress state. The test temperatures $\mathrm{T}_{1}, \mathrm{~T}_{2}, \mathrm{~T}_{3}$ and $\mathrm{T}_{4}$ are indicated by arrows.

Fig.2(a-d) show the stress-strain curves taken at $T_{1}, T_{2}, T_{3}$ and $T_{4}$, respectively. Fig.3(a-d) show the normalized $R / R_{0}$ electric resistance (where $R_{0}$ is the $E R$ at $\epsilon=0$ ) versus strain curves detected during the tensile tests of fig.2(a-d).

\section{4.-Discussion}

Fig.3(a,b), corresponding to specimens in full martensitic phase, show that $E R$ has a linear trend as a function of strain, independent upon either elastic or variant re-orientation domain. The smooth transition from elastic deformation domain of thermal martensite to variant reorientation domain ( $f i g .2(a, b)$ ), does not noticeably affect ER.

The ER linear dependence can be explained as follows: lacking an electronic band structure change due to a phase transition, ER depends upon geometrical factors through lenght/section changes under uniaxial loading. However, geometrical factors do not explain the slight different slope found at the test temperatures $T_{1}$ and $T_{2}$, in full martensitic phase.

Deformation in full B2 phase ( $f i g .2 d$ ), induces a change in slope for ER, shown in fig.3d, mainly due to the resistivity change associated with the stress-induced martensite(SIM): both in the B2 elastic deformation domain and on the pseudoelastic plateau an overall linear dependence is exhibited, at least up to nearly 38 strain where sIM elastic deformation domain is probably set up. 

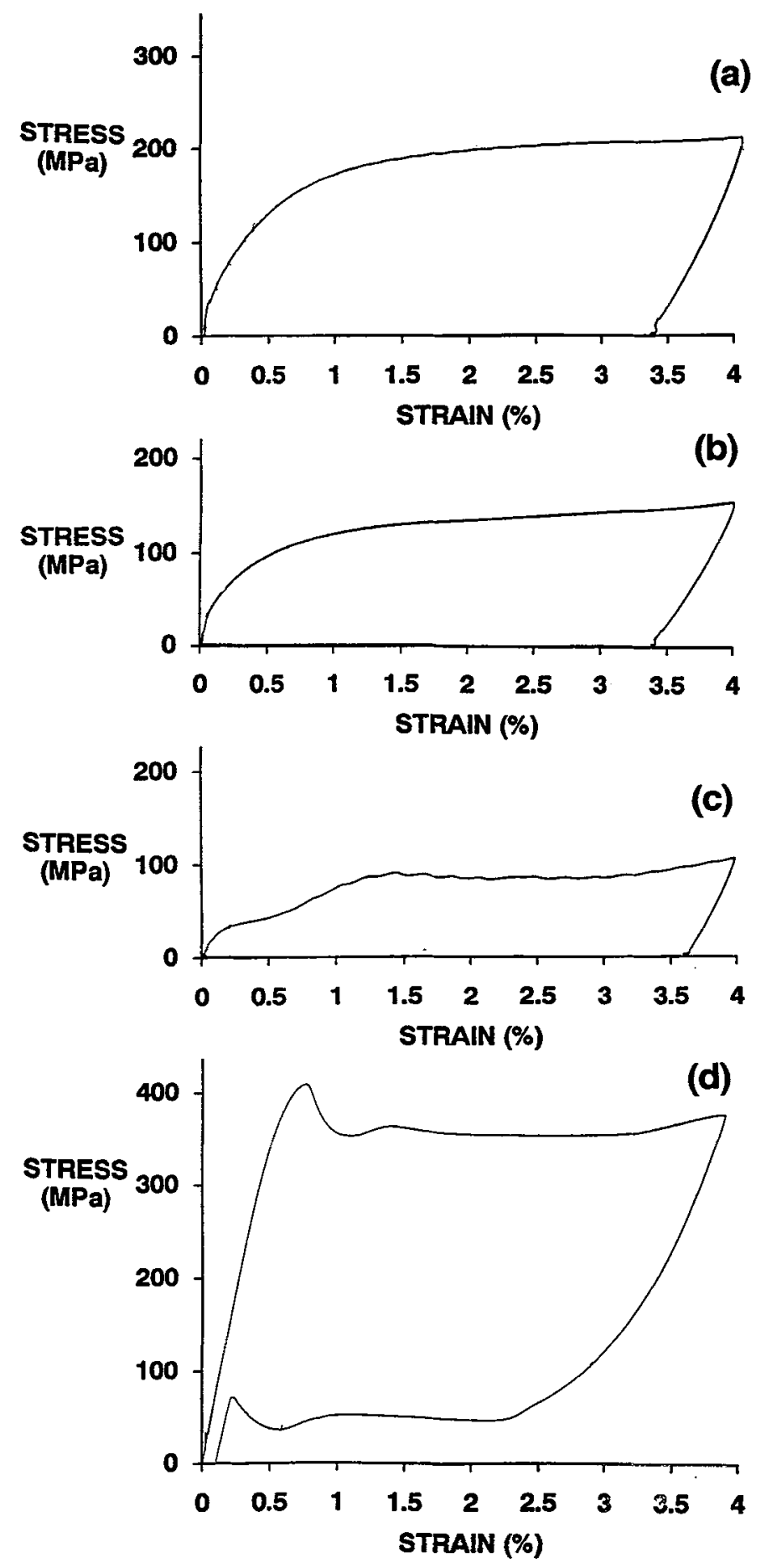

Fig.2 - stress vs. Strain curves of the examined NiTi specimens at the test temperatures $T_{1}(a), T_{2}(b), T_{3}(c)$ and $T_{4}$ (d). 


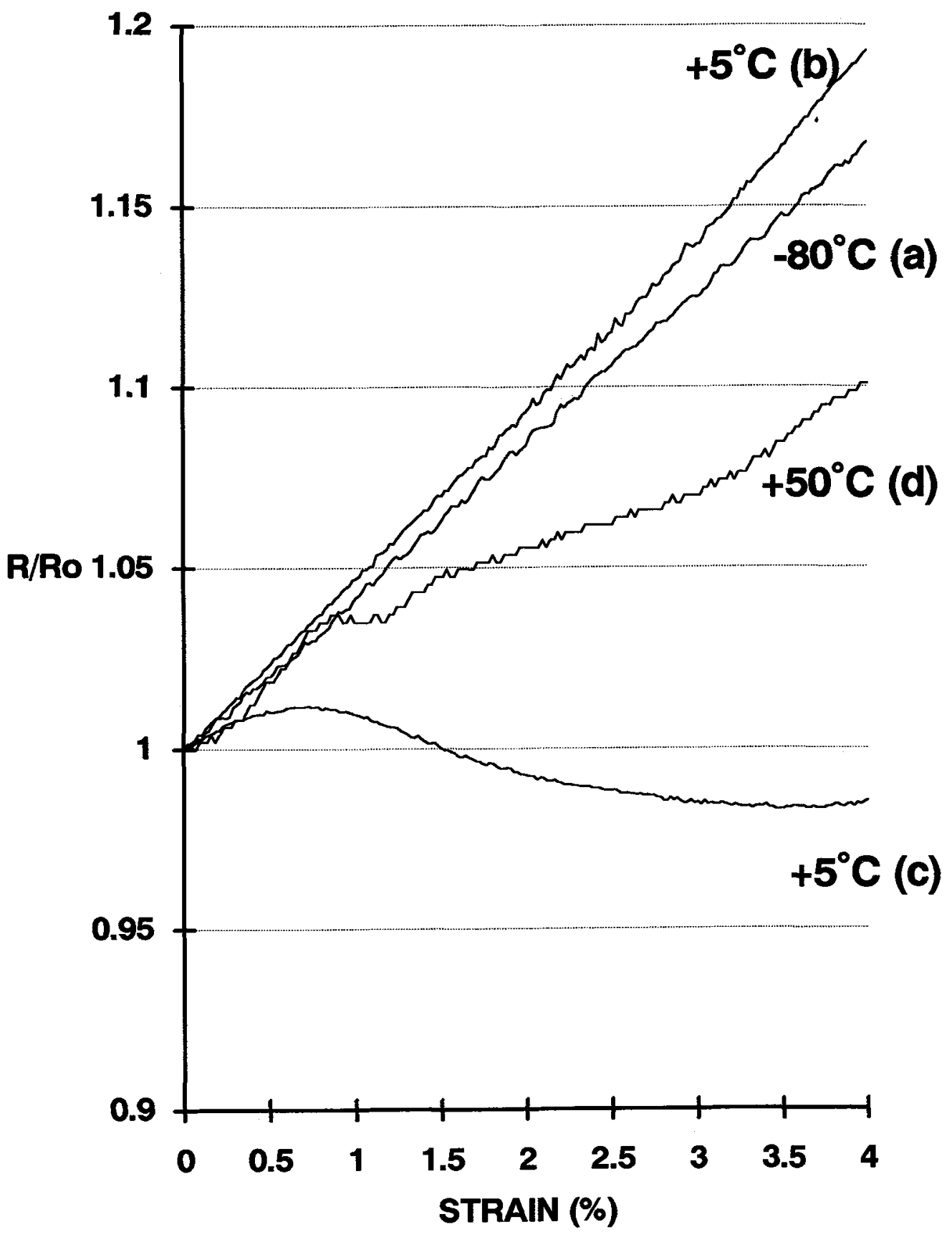

Fig.3 - Normalized $R / R_{0}\left(R_{0}\right.$ is the $E R$ at $\left.\epsilon=0\right)$ Electric Resistance vs. strain curves of the examined NiTi specimens at the test temperatures $T_{1}(a), T_{2}(b), T_{3}(c)$ and $T_{4}$ (d), detected during tensile tests of fig.2(a-d). 
Deformation in R-phase (fig.2c), within the hysteresis cycle, gives rise to a non-linear behaviour for $E R$, shown in fig.3c: a very small $R / R_{0}$ change is present in comparison with the other examined cases. Probably opposite contributions, nearly equal in entity, balance each other: on one side, geometrical factors promote an increase of $R / R_{\circ}$ of about 15-20\% per 48 strain; on the other side, the re-orientation of $R$-phase followed by SIM, promotes a decrease in $R / R_{0}$ due to the phase transformation, of about $20 \%$ as expected for the $R-M$ transition at the test temperature (fig.1).

\section{5.-Conclusions}

ER measurements have been here detected simultaneously to stressstrain tensile tests, performed on NiTi specimens in one start single phase, either martensitic B19' or parent B2 phase.

In full $B 19^{\prime}$ phase $\left(T=T_{1}, T_{2}\right), E R$ shows an overall linear dependence upon strain: since an electronic band structure change, due to a phase transition, is not involved, geometrical factors are mainly responsible of the linear behaviour, even if the slightly different slope observed at the test temperature $T_{1}$ and $T_{2}$ is not explained.

In full B2 phase $\left(T=T_{4}\right)$, in presence of a stress-induced phase transition, both geometrical factors and electronic band structure change modify ER as a function of strain: nevertheless, both in the B2 elastic deformation domain and on the pseudoelastic plateau an overall distinct inear dependence is exhibited.

In $R$-phase $\left(T=T_{3}\right)$, a small non-linear change for ER vs. strain is found. The stress-induced phase transition from the higher electric resistivity R-phase to the lower resistivity M-phase balances geometrical factors contributions.

$E R$ as a function of strain seems therefore apt to implement investigations on the macroscopic stress-strain behaviour: detected simultaneously, they allow to monitor both macro and micro-structural changes in shape memory alloys.

\section{Ackwoledgements}

The work has been partially supported by C.N.R.-P.F. MSTA project. One of the Authors (G.A.) acknowledges the support of the Italian Board of Education and Research (MURST).

\section{6. -References}

1) MIYAZAKI, S, OTSUKA, K, ISIJ 29 (1989) 353.

2) GOO,E, SINCLAIR,R, Acta Metall, 33 (1985) 1717.

3) MIYAZAKI, S, OTSUKA, K, Met.Trans. 17A (1986) 53. 